\title{
Measuring the Optical Point Spread Function of FACT Using the Cherenkov Camera
}

\author{
M. Noethe ${ }^{*}$, J. Adam $^{b}$, M. L. Ahnen ${ }^{a}$, D. Baack ${ }^{b}$, M. Balbo ${ }^{c}$, A. Biland ${ }^{a}$, M. Blank ${ }^{d}$,

 \\ D. Elsaesser ${ }^{b}$, C. Hempfling ${ }^{d}$, T. Herbst $^{d}$, D. Hildebrand ${ }^{a}$, L. Kortmann ${ }^{b}$, \\ L. Linhoff ${ }^{b}$, M. Mahlke ${ }^{a}$, K. Mannheim ${ }^{d}$, S. A. Mueller ${ }^{a}$, D. Neise ${ }^{a}$, A. Neronov ${ }^{c}$, \\ J. Oberkirch ${ }^{b}$, A. Paravac ${ }^{d}$, F. Pauss ${ }^{a}$, W. Rhode ${ }^{b}$, B. Schleicher ${ }^{d}$, F. Schulz ${ }^{b}$,

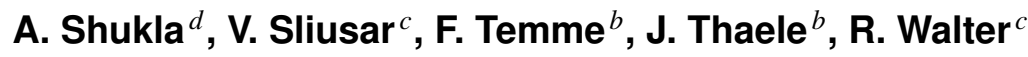 \\ Email: maximilian.noethe@tu-dortmund.de \\ ${ }^{a}$ ETH Zurich, Institute for Particle Physics \\ Otto-Stern-Weg 5, 8093 Zurich, Switzerland \\ ${ }^{b}$ TU Dortmund, Experimental Physics 5 \\ Otto-Hahn-Str. 4, 44221 Dortmund, Germany \\ ${ }^{c}$ University of Geneva, ISDC Data Center for Astrophysics \\ Chemin d'Ecogia 16, 1290 Versoix, Switzerland \\ ${ }^{d}$ Universität Würzburg, Institute for Theoretical Physics and Astrophysics \\ Emil-Fischer-Str. 31, 97074 Würzburg, Germany
}

FACT, the First G-APD Cherenkov Telescope, is an Imaging Air Cherenkov Telescope (IACT) operating since 2011 at the Observatorio del Roque de los Muchachos on the Canary Island of La Palma. As typical for IACTs, its reflector is comprised of smaller mirror facets and not protected by a dome. In the case of FACT, 30 hexagonal facets form a total mirror area of $9.5 \mathrm{~m}^{2}$. Hence, it is crucial to monitor the optical properties of this system and realign the facets if necessary. Up to now, measuring the Point Spread Function of FACT required human interaction to mount a screen and an optical camera. In this contribution, a new method to measure the optical Point Spread Function using directly the Cherenkov camera of the telescope is presented. Inspired by the method radio telescopes use to determine their resolution, the telescope is pointed towards a fixed position on the trajectory of a star. During the star's passage through the field of view, the camera is read out using a fixed rate. In each event, the pedestal variance is determined for each pixel. This value is directly correlated with the amount of night sky background light a pixel received. Translating the time of the measurement to the position of the star in the camera enables to determine the optical point spread function from this measurement. As the measurement is done for each pixel along the trajectory of the star, the Point Spread Function can be determined not only for the camera center but for the entire field of view. In this contribution, the new method will also be compared with the existing methods of determining the optical Point Spread Function: direct measurement with an optical camera and the width of Muon ring events.

35th International Cosmic Ray Conference - ICRC2017 -

10-20 July, 2017

Bexco, Busan, Korea

* Speaker. 


\section{Introduction}

FACT (First G-APD Cherenkov Telescope) [1] is the first Imaging Air Cherenkov Telescope which uses Silicon Photomultipliers (SIPMs) as photo sensors. Observing since first light in October 2011 at the Observatorio del Roque de los Muchachos on the Canary Island of La Palma (Spain), the main goal of FACT is the monitoring of very high energy gamma-ray sources in the Northern hemisphere.

The camera consists of 1440 pixels and has a diameter of approximately $40 \mathrm{~cm}$, resulting in a field of view of $4.5^{\circ}$.

FACT is using a segmented mirror that is comprising 30 hexagonal facets with a total mirror area of $9.51 \mathrm{~m}^{2}$ and a focal length of $4.9 \mathrm{~m}$. The facets are oriented in a hybrid between a parabolic and a Davies-Cotton-geometry [2]. Due to the segmented mirror, monitoring the alignment of the facets is important to estimate if and when a new mirror justage might be necessary.

In this contribution, the first results of applying a technique inspired by radio telescopes is presented. The telescope is pointed to a fixed position in the sky on the trajectory of a star. During the passage of the star through the field of view, the Cherenkov camera is read out using a fixed rate of $200 \mathrm{~Hz}$. For each recorded event, the so called pedestal variance is calculated in each pixel. This number is directly correlated with the amount of star light the pixel received. The observation time of the event is then translated into the distance the star traveled through the camera. To get a first estimate of the point spread function, the pedestal variance vs. travel length is smoothed using a low-pass filter and then fit with a gaussian distribution. The standard deviation of the gaussian is used as a measure for the width of the point spread function.

\section{Pedestal Variance}

Cherenkov photons from extensive air showers arrive in the camera in very short bursts resulting in voltage pulses, where the area under the pulse can be used to estimate the number of photons. The FACT camera uses AC-coupling to keep the voltage baseline at 0 . This makes it impossible to directly estimate the steady stream of night sky background photons from the amplitude of the voltage time series. Instead, the fluctuation of the voltage time series is used to estimate the night sky contribution in each pixel. The time series are split into divisions as long as the integration window that is used to estimate the number of Cherenkov photons, and is integrated in each of theses windows. Then the variance over these integrals is calculated, the resulting number is called the pedestal variance. Figure 1 shows the mean pedestal variance for different simulated night sky brightnesses. The pedestal variance has a linear dependency with the amount of background photons and thus can directly be used as a measure of night sky brightness. 




Figure 1: Mean pedestal variance vs. simulated night sky background photon rate for simulated random trigger events.

\section{Star Passage Measurement}

The measurements analysed in this contribution were taken on 17.02.2017 using the magnitude 2.78 star Cursa, which at a declination of $-5^{\circ}$ is close to the celestial equator. [3] This results in the star passaging through the camera in a straight line, as can be seen in Figure 2, which shows the maximum pedestal variance in each pixel over the duration of the measuremt of $25 \mathrm{~min}$. The telescope was pointed to a zenith distance of $34.2^{\circ}$ and an azimuth of $190^{\circ}$, so that the star would pass right through the camera center in the middle of the measurement.

During the star passage, the camera was read out at a fixed rate of $200 \mathrm{~Hz}$ using a region of interest of 300 voltage values corresponding to $150 \mathrm{~ns}$.

For each recored event, the pedestal variance in each pixel is calculated using an integration window of 30 values. The measurment time of each event is translated to the corresponding distance the star moved in the camera plane. To get an estimate of the point spread function in each pixel, the pedestal variance time series is smoothed using a low-pass filter and then fit with a gaussian function plus a constant baseline.

Two exemplary pedestal variance curves with the corresponding fit results can be seen in Figure 3. All fit results for the pixels where cursa passed trough are shown in the camera view in Figure 4. Over the full star passage, the standard deviation of the gaussian is estimated to $(6.25 \pm 0.46) \mathrm{mm}$. 


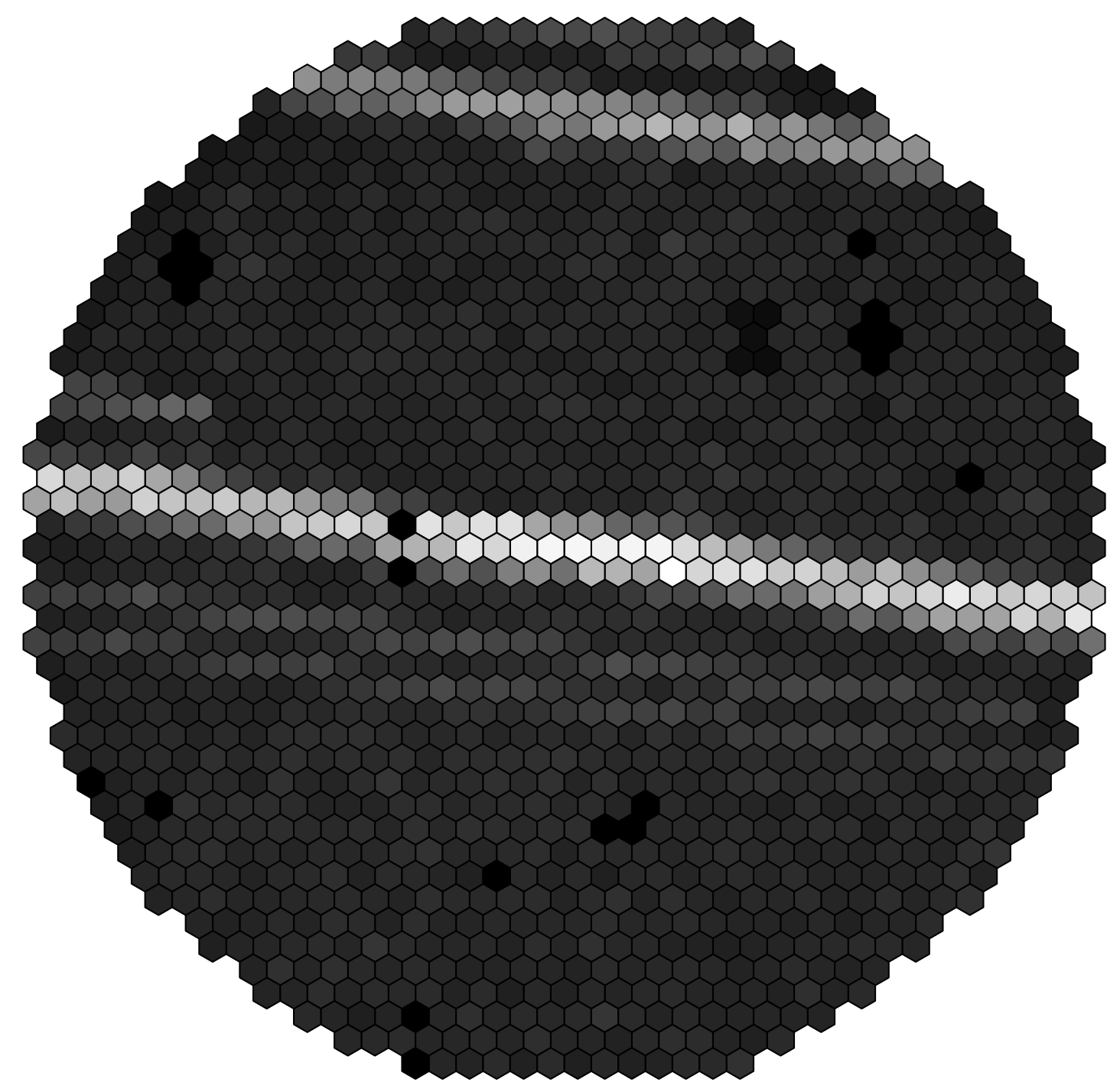

Figure 2: Maximum of the smoothed pedestal variance over the time of the measurement. 




Figure 3: Smoothed pedestal variance for pixels 0 and 10 with gaussian fits.
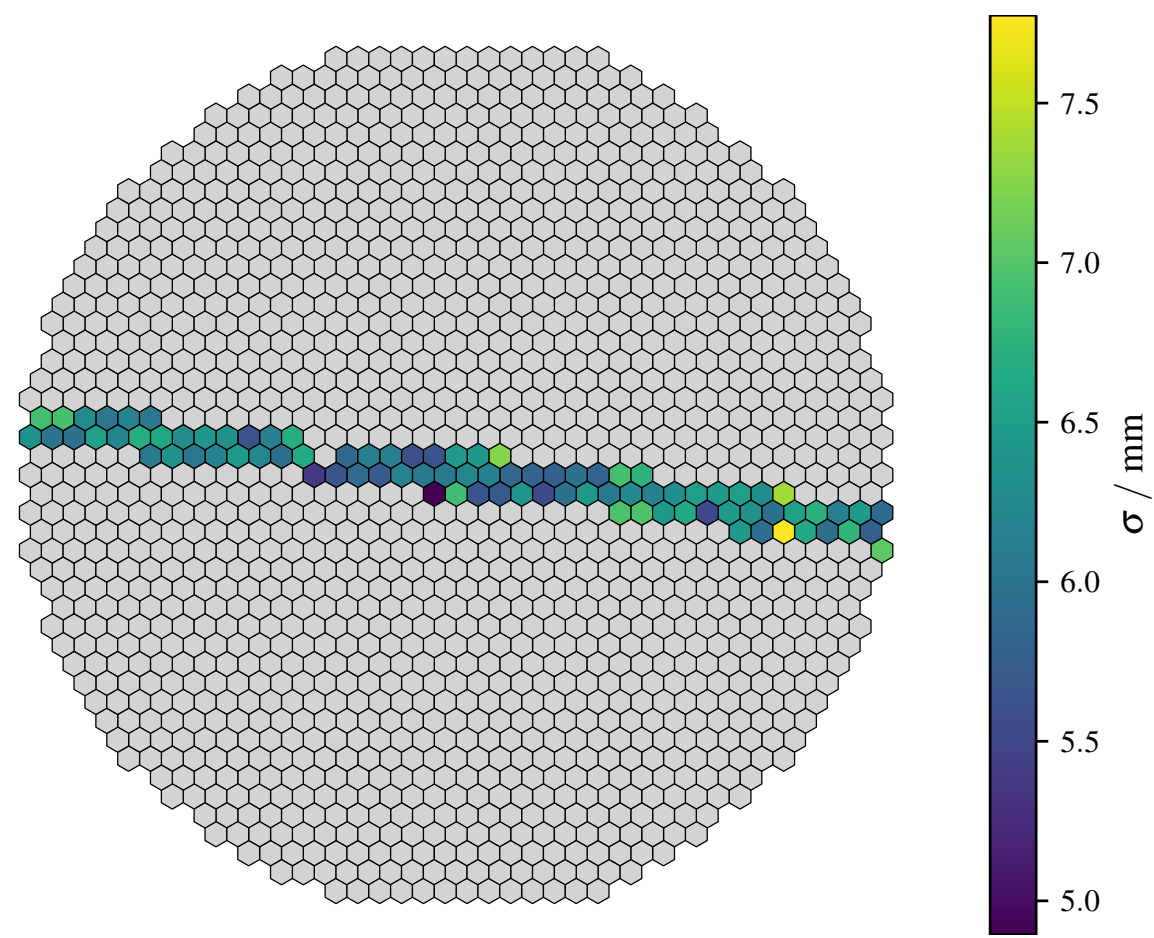

Figure 4: Camera overview of the successful fits to the pixels where Cursa passed through. 


\section{Conclusions}

A novel method to estimate the optical resolution of Cherenkov telescopes using the main image sensor has been introduced. The first results are promising but the technique has to be evaluated further. The influence of the pixel shape and orientation towards the star trajectory has to be taken into account, to be completely independent of the pixels of the camera. Taking multiple star passages in different directions could be used to reconstruct a full two-dimensional point spread function. In the future, the method might be used to monitor the optical resolution of FACT, as it is currently the only method not requiring human interaction at the telescope. The needed time to measure the point spread function can be reduced by not taking a full passage through the camera but only of a few central pixels.

Acknowledgment The important contributions from ETH Zurich grants ETH-10.08-2 and ETH-27.12-1 as well as the funding by the Swiss SNF and the German BMBF (Verbundforschung Astro- und Astroteilchenphysik) and HAP (Helmoltz Alliance for Astroparticle Physics) are gratefully acknowledged. Part of this work is supported by Deutsche Forschungsgemeinschaft (DFG) within the Collaborative Research Center SFB 876 "Providing Information by Resource-Constrained Analysis", project C3. We are thankful for the very valuable contributions from E. Lorenz, D. Renker and G. Viertel during the early phase of the project. We thank the Instituto de Astrofísica de Canarias for allowing us to operate the telescope at the Observatorio del Roque de los Muchachos in La Palma, the Max-Planck-Institut für Physik for providing us with the mount of the former HEGRA CT3 telescope, and the MAGIC collaboration for their support

\section{References}

[1] H. Anderhub et al. "Design and operation of FACT - the first G-APD Cherenkov telescope". In: JINST 8.06 (2013), P06008.

[2] M. Ahnen et al. "Bokeh mirror alignment for Cherenkov telescopes". In: Astroparticle Physics 82 (2016), pp. 1-9.

[3] M. A. C. Perryman et al. "The HIPPARCOS Catalogue". In: Astronomy and Astrophysics 323 (July 1997), pp. L49-L52. 